\title{
GENERATING COMBINATORIAL COMPLEXES OF POLYHEDRAL TYPE
}

\author{
EGON SCHULTE
}

\begin{abstract}
The paper describes a method for generating combinatorial complexes of polyhedral type. Building blocks $\mathbf{B}$ are implanted into the maximal simplices of a simplicial complex $\mathbf{C}$, on which a group operates as a combinatorial reflection group. Of particular interest is the case where $\mathbf{B}$ is a polyhedral block and $\mathbf{C}$ the barycentric subdivision of a regular incidence-polytope $\mathbf{K}$ together with the action of the automorphism group of $\mathbf{K}$.
\end{abstract}

1. Introduction. In this paper we discuss a method for generating certain types of combinatorial complexes. We generalize ideas which were recently applied in [23] for the construction of tilings of the Euclidean 3-space $E^{3}$ by dodecahedra.

The 120-cell $\{5,3,3\}$ in the Euclidean 4-space $E^{4}$ is a regular convex polytope whose facets and vertex-figures are dodecahedra and 3-simplices, respectively (cf. Coxeter [6], Fejes Tóth [12]). When the 120-cell is centrally projected from some vertex onto the 3 -simplex $T$ spanned by the neighboured vertices, then a dissection of $T$ into dodecahedra arises (an example of a central projection is shown in Figure 1). This dissection can be used as a building block for a tiling of $E^{3}$ by dodecahedra. In fact, in the barycentric subdivision of the regular tessellation $\{4,3,4\}$ of $E^{3}$ by cubes each 3 -simplex can serve as as a fundamental region for the symmetry group $W$ of $\{4,3,4\}$ (cf. Coxeter [6]). Therefore, mapping $T$ affinely onto any of these 3 -simplices and applying all symmetries in $W$ turns the dissection of $T$ into a tiling $\mathbf{T}$ of the whole space $E^{3}$. In particular, $\mathbf{T}$ has the face-to-face property meaning that the intersection of any two dodecahedra in $\mathbf{T}$ is either empty or a face of each. This is due to the fact that the symmetry group $W$ of $\{4,3,4\}$ is generated by the reflections in the hyperplanes bounding one fundamental region. Moreover, the tiling $\mathbf{T}$ has only 116 isometric prototiles (one for each dodecahedra in the dissection of $T$ ), meaning that every dodecahedron on $\mathbf{T}$ is congruent to one dedecahedron in a set of 116 dodecahedra.

In a similar fashion we can also tile each regular 3-polytope by dodecahedra, again making use of the fact that its symmetry group is generated by reflections in hyperplanes bounding one fundamental region (cf. Coxeter [6]). Also, there is no need to restrict our considerations to dimension 3 and dodecahedra as tiles. For instance, the construction works equally well for any convex $(d-1)$-polytope $P$ that is the facet-type of an equifacetted convex $d$-polytope $Q$ with at least one $d$-valent vertex.

These geometrical constructions will be generalized in a combinatorial sense providing monotypic combinatorial complexes, that is, complexes whose facets are

Received by the editors April 20, 1984.

1980 Mathematics Subject Classification (1985 Revision). Primary 51M20; Secondary 05B45. 


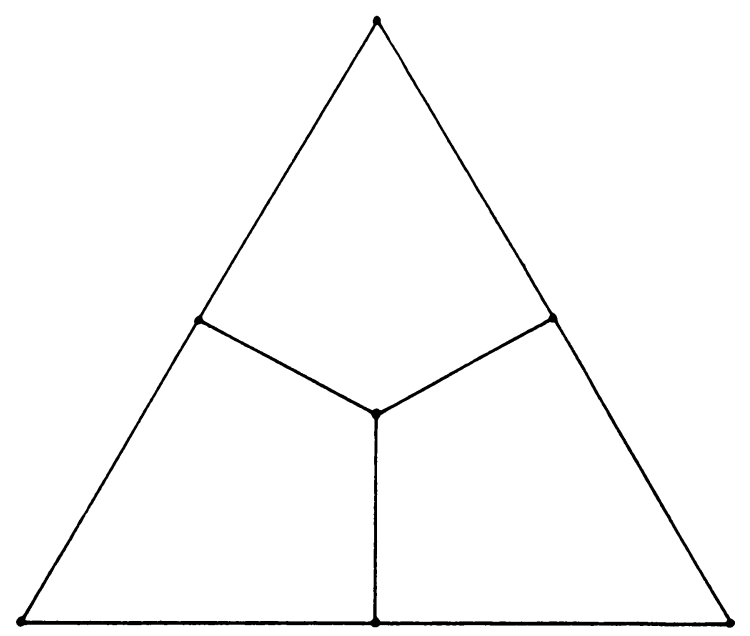

FigURE 1. A projection of the 3-cube (centrally from a vertex $x$ ) onto the 2 -simplex (spanned by the vertices neighboring $x$ )

all of the same combinatorial type. Again, our building blocks will be complexes whose 'carrier' are simplices. These buildings blocks will be implanted into simplices that are fundamental regions for certain groups operating on a simplical complex and generated by 'combinatorial reflections' in the walls of one fundamental region. Then, the group-action turns the simplicial complex into a complex whose facets are of the same combinatorial type as the facets of the building block. Moreover, the group operates on the complex, and each transitivity class of the group action on the facets of the complex is represented by exactly one facet in the building block.

The most interesting examples arise when the group is the automorphism group of a regular incidence-polytope $\mathbf{K}$ operating on the barycentric subdivision of $\mathbf{K}$ (cf. [21]). These complexes admit also geometrical realizations in Euclidean spaces of small dimension. As a special case we obtain the above-mentioned tilings of the Euclidean space.

The first sections will contain a summary of the necessary definitions. For notation and basic results the reader is referred to Grünbaum [13] and McMullenShephard [18] for convex polytopes, to Grünbaum-Shephard [15] for tilings, and to Danzer-Schulte [10] for regular incidence-complexes. Our main construction is described in $\S 6$. $\S 7$ contains some results about geometrical realizations of our complexes.

2. Complexes. We begin by introducing the notion of a complex. It is somewhat analogous to Danzer's notion of an incidence-complex (cf. [10]) and Grünbaum's notion of a polystroma (cf. [14]).

In this paper we mean by a complex of dimension $d$ (or briefly a $d$-complex) a partially ordered set $(\mathbf{K}, \leq)$ that is defined by the properties (I1)-(I3).

(I1) There are elements $F_{-1}$ and $F_{d}$ such that $F_{-1} \leq F \leq F_{d}$ for all $F$ in $\mathbf{K}$.

(I2) Every totally ordered subset of $\mathbf{K}$ is contained in a totally ordered subset with exactly $d+2$ elements, a so-called flag. 
The elements of $\mathbf{K}$ are called faces. For convience, we will not distinguish a face $F$ and the section-complex $\{G \mid G \leq F\}$ of faces which are majorized by $F$. With every face $F$ we can associate a number $\operatorname{dim}(F)$ called the dimension of $F$, where $\operatorname{dim}(F)+2$ is the cardinality of the flags in $\{G \mid G \leq F\}$. In particular, $\operatorname{dim}\left(F_{-1}\right)=-1$ and $\operatorname{dim}\left(F_{d}\right)=d$. We call $F$ a vertex, an edge, an $i$-face or a facet, if $\operatorname{dim}(F)=0,1, i$ or $d-1$, respectively. The section-complex $\{G \mid G \geq F\}$ of all faces that are greater than or equal to $F$ is called the coface to $F$, and a vertex-figure in case $F$ is a vertex.

Our last defining property is

(I3) $K$ is connected, that means: if $f$ and $g$ are two different flags of $K$ and $h:=f \cap g$, then there is a finite sequence of flags $f=f_{1}, f_{2}, \ldots, f_{n-1}, f_{n}=g$, all containing $h$, such that $f_{m+1}$ differs from $f_{m}$ in exactly one face $(1 \leq m \leq n-1)$.

Note that the word 'complex' was used in a slightly different sense in [10, 21], where it abbreviates the word 'incidence-complex'. Incidence-complexes are special types of complexes that satisfy not only (I1)-(I3) but also (I4).

There are cardinal numbers $k_{0}, k_{1}, \ldots, k_{d-1} \geq 2$ with the following property. For any two faces $F$ and $G$ with $F<G$ and $\operatorname{dim}(F)+1=$ $i=\operatorname{dim}(G)-1$, there are exactly $k_{i} i$-faces $H$ in $\mathbf{K}$ with $F<H<G$ $(i=0,1, \ldots, d-1)$.

An incidence-complex is called homogeneous of order $k-1$, if $k_{i}=k$ for all $i$ (cf. Danzer [9]). The homogeneous incidence-complexes of order 1 are called incidence-polytopes; then $k_{i}=2$ for all $i$.

For many of our complexes the numbers $k_{0}, \ldots, k_{d-2}$ will also exist and will be 2. However, in general, the number of facets that surround a given $(d-2)$-face $F$ of $\mathbf{K}$ will depend on $F$. We denote this number $k_{d-1}(F)$, or $k_{d-1}(\mathbf{K}, F)$.

In most cases we are concerned with nondegenerate complexes. A complex $\mathbf{K}$ is called nondegenerate, if the partial order induces a lattice on $\mathbf{K}$. Otherwise we call $\mathbf{K}$ degenerate. Nondegeneracy is exactly the combinatorial analogue of the face-to-face property for tilings.

There are also a combinatorial analogue for so-called montypic tilings (cf. Grünbaum-Mani-Shephard $[\mathbf{1 6}] ;[\mathbf{2 3}])$. The investigation of such tilings was initiated by a problem posed by L. Danzer in 1975: given a convex 3-polytope $P$, is there a locally finite face-to-face tiling of $E^{3}$ by convex polytopes isomorphic to $P$ ? (cf. Danzer-Grünbaum-Shephard [11], Larman-Rogers [17]). Counterexamples were recently discovered by the author in $[\mathbf{2 2}]$ and $[\mathbf{2 3}]$.

Let $\mathbf{L}$ be a $(d-1)$-complex. By a monotypic complex of type $\mathbf{L}$ we mean a $d$-complex $\mathbf{K}$ whose facets are isomorphic to $\mathbf{L}$. If $\mathbf{L}$ is the face-lattice of a convex $(d-1)$-polytope $P$, then we simply say that $\mathbf{K}$ is monotypic of type $P$.

In the sequel, we will also use the notion of a regular incidence-complex. An incidence-complex $\mathbf{K}$ is named regular, if its group $A(\mathbf{K})$ of combinatorial automorphisms of $\mathbf{K}$ (that is, of incidence-preserving permutations) is flag-transitive.

Of particular interest will be the case where $\mathbf{K}$ is a regular incidence-polytope. We say that a regular incidence-polytope is of type $\left\{p_{1}, p_{2}, \ldots, p_{d-1}\right\}$, if the sectioncomplexes $\{H \mid F \leq H \leq G\}$ belonging to an $(i-2)$-face $F$ and an $(i+1)$-face $G$ incident with $F$ are isomorphic to the face-lattice of usual $p_{i}$-gons in the plane for $i=1, \ldots, d-1$. 
For an introduction to the theory of incidence-complexes the reader is referred to [10]. Many considerations extend to our complexes. Later, we will also apply the results of $[\mathbf{2 1}]$, which analyze the group of regular incidence-complexes.

3. Simplicial complexes. In this section we describe the simplicial complexes, which replace the barycentric subdivision in the construction of dodecahedral tilings of the Euclidean 3-space. They are provided by certain groups, or more exactly, by systems of generators for these groups. These generators can be regarded as 'combinatorial reflections' in the walls of one of the maximal simplices of the complex, and this simplex turns out to be a fundamental region for the group. If the group happens to be the symmetry group of the regular tessellation $\{4,3,4\}$ of $E^{3}$ by cubes, then we get a simplicial complex isomorphic to the barycentric subdivision of $\{4,3,4\}$. Analogous results hold for all regular polytopes and honeycombs, and all regular complex polytopes (cf. Coxeter $[\mathbf{5}, \mathbf{6}, \mathbf{7}]$ ).

Let $U$ be a group and $D$ an index set of cardinality $m$. For each $i$ in $D$ let $R_{i}$ be a subgroup of $U$, and let $U$ be generated by these subgroups, that is, $U=\left\langle R_{i} \mid i \in D\right\rangle$. For every nonempty subset $I$ of $D$ define $U_{I}:=\left\langle R_{i} \mid i \in I\right\rangle$, so that in particular $U_{D}=U$ and $U_{\{i\}}=R_{i}$ for all $i$ in $D$. Moreover, let $U_{\varnothing}=\bigcap_{i \in D} R_{i}$. For the subgroups $U_{I}$ we require the following intersection property.

$$
\text { If } I, J \subset D \text {, then } U_{I} \cap U_{J}=U_{I \cap J} \text {. }
$$

Following Tits $\left[\mathbf{2 5}\right.$, p. 5] this system of subgroups $U_{I}$ can serve for the construction of a simplicial complex, whose simplices are just the left cosets of the $U_{I}$ in $U$ (in Tits's notation, $G^{i}=U_{D \backslash\{i\}}$ for each $i$ in $D$ ). In the sequel, we will always assume that the subgroups $U_{I}$ are pairwise different. In particular this implies that the subgroups $R_{i}$ are pairwise different and also different from $U_{\varnothing}$.

By $\mathbf{C}=\mathbf{C}(U)$ (or more exactly, $\mathbf{C}\left(U ; R_{i}, i \in D\right)$ ) we denote the set of all left cosets of the subgroups $U_{I}$ for $I \subset D$, that is,

$$
\mathbf{C}=\mathbf{C}(U)=\left\{\varphi U_{I} \mid I \subset D, \varphi \in U\right\} .
$$

In $\mathbf{C}$ we introduce the following partial order. For two elements $\varphi U_{I}$ and $\psi U_{J}$, we set $\varphi U_{I} \leq \psi U_{J}$, if and only if $J \subset I$ and $\psi U_{J} \subset \varphi U_{I}$ in the set-theoretical sense. Since all the $U_{I}$ are different, this simply means that $\leq$ is the opposite of the set-theoretical inclusion.

It can be shown that $\mathbf{C}$ endowed with this partial order is a simplicial complex (in the usual sense), whose maximal simplices have dimension $m-1$. They are just the cosets of $U_{\varphi}$ in $U$, and the faces of the $(m-1)$-simplex $\varphi U_{\varnothing}$ are the $(m-1-|I|)$ simplices $\varphi U_{I}$ for $I \subset D$; in particular, the $(m-2)$-faces and vertices of $\varphi U_{\varnothing}$ are the cosets $\varphi U_{\{i\}}$ and $\varphi U_{D \backslash\{i\}}$ for $i$ in $D$, respectively.

Furthermore, the simplex $\varphi U_{I}$ is a face of the simplex $\psi U_{J}$, if and only if $J \subset I$ and $\varphi^{-1} \psi \in U_{I}$. This shows in particular that the coface to the face $U_{I}$ in $\mathbf{C}$ is isomorphic to the simplicial complex $\mathbf{C}\left(U_{I}\right)$ belonging to the generators $R_{i}$ for $i$ in $I$; it consists of the faces $\psi U_{J}$ with $J \subset I$ and $\psi \in U_{I}$.

The group $U$ operates on $\mathbf{C}$ in a natural way. Each element $\tau$ of $U$ induces the automorphism $\tilde{\tau}: \mathbf{C} \rightarrow \mathbf{C}$ defined by $\tilde{\tau}\left(\varphi U_{I}\right):=\tau \varphi U_{I}$ for $\varphi \in U$ and $I \subset D$. Here, $\tilde{\tau}$ is the identity automorphism, if and only if $\tau \in \varphi^{-1} U_{\varnothing \varphi}$ for all $\varphi$ in $U$. Clearly, for each $I \subset D, U$ operates transitively on the $(m-1-|I|)$-faces $\varphi U_{I}$ for $\varphi \in U$, 
so that, in particular, $U$ is transitive on the $(d-1)$-simplices of $\mathbf{C}$. The stabilizer of the face $U_{I}$ of $\mathbf{C}$ in $U$ is just the group $U_{I}$ itself.

For each $i$ in $D$, the $(m-1)$-simplices surrounding the $(m-2)$-face $U_{\{i\}}=R_{i}$ of $U_{\varnothing}$ are just the cosets $\rho U_{\varnothing}$ with $\rho$ in $R_{i}$. Therefore, in a sense, the elements of $R_{i}$ can be interpreted as 'combinatorial reflections' in the wall $U_{\{i\}}$ of $U_{\varnothing}$.

As an immediate consequence we see that two $(m-1)$-simplices $\varphi U_{\varnothing}$ and $\psi U_{\varnothing}$ share an $(m-2)$-face, if and only if $\varphi=\psi \rho$ for an element $\rho$ in some $R_{i}$. Since $U$ is generated by the groups $R_{i}$, this implies that any two $(m-1)$-simplices can be joined by a chain of $(m-1)$-simplices where any two consecutive members share an $(m-2)$-face.

Taking into account the structure of the cofaces of $\mathbf{C}$ this property implies the property (I3) for $\mathbf{C}$, so that $\mathbf{C}$ turns out to be a nondegenerate $m$-complex in the sense of $\S 2$ (with $U$ as the (-1)-face and some suitably adjoined $d$-face). In particular, for $\varphi$ in $U$,

$$
k_{m-1}\left(\varphi U_{\{i\}}\right)=k_{m-1}\left(U_{\{i\}}\right)=\left|U_{\{i\}}: U_{\varnothing}\right|=\left|R_{i}: U_{\varnothing}\right| \geq 2 .
$$

Finally, we can show that the $(m-1)$-simplex $U_{\varnothing}$ together with its faces $U_{I}$, $I \subset D$, can be regarded as a fundamental region in $\mathbf{C}$ for the group $U$. In fact, any face of $\mathbf{C}$ has an equivalent face in $U_{\varnothing}$, and any two equivalent faces of $U_{\varnothing}$ coincide.

4. Regular incidence-complexes. It is common practice to associate with a partially ordered set the simplicial complex of its finite totally ordered subsets. In this section we describe the simplicial complexes associated with regular incidencecomplexes. In particular, we make use of the results in [21].

Let $\mathbf{K}$ be a regular $d$-incidence-complex whose $(-1)$ - and $d$-faces are $F_{-1}$ and $F_{d}$, respectively. The barycentric subdivision of $\mathbf{K}$ is defined as the simplicial complex

$$
\mathbf{C}(\mathbf{K}):=\left\{g \mid g \subset \mathbf{K} \text { totally ordered, and } F_{-1}, F_{d} \notin g\right\} .
$$

The maximal simplices (of dimension $d-1$ ) are in one-to-one correspondence with the flags of $\mathbf{K}$, and their faces are given by the subsets of the respective flag. In order to turn $\mathbf{C}(\mathbf{K})$ into a $d$-complex in the sense of $\S 2$ we adjoin any element as the $d$-face; note the $(-1)$-face is the empty set.

It has been proved in $[\mathbf{2 1}, \S 7]$ that the barycentric subdivision $\mathbf{C}(\mathbf{K})$ of a regular incidence-complex $\mathbf{K}$ is isomorphic to the simplicial complex $\mathbf{C}(U)$ belonging to its automorphism group $U=A(\mathbf{K})$ (or any flag-transitive subgroup). The isomorphism is obtained as follows.

Let $f=\left\{F_{-1}, F_{0}, F_{1}, \ldots, F_{d}\right\}$ be a fixed flag in $\mathbf{K}$, where $F_{i}$ denotes the $i$-face of $f(i=-1,0, \ldots, d)$. For $i=-1,0, \ldots, d$, let $R_{i}$ be the stablizer of $f \backslash\left\{F_{i}\right\}$ in $U=A(\mathbf{K})$. Then, $R_{-1} \subset R_{i}$ for all $i$, and one can show (cf. [21, §2]) that the automorphism group $U$ and its subgroups $R_{0}, \ldots, R_{d-1}$ have all the required properties of $\S 3$ (with $D=\{0, \ldots, d-1\}, m=d$ ). In particular, $U=\left\langle R_{0}, \ldots, R_{d-1}\right\rangle$, and $U_{\varnothing}=R_{-1}=R_{d}$ is the stablizer of the flag $f$ in $U$. More generally, if $I \subset\{0, \ldots, d-1\}=D$, then the stabilizer of the subset $C_{I}:=f \backslash\left\{F_{i} \mid i \in I\right.$ or $i=-1, d\}$ of $f$ is just the subgroup $U_{I}=\left\langle R_{i} \mid i \in I\right\rangle$ of $U$.

By the transitivity properties of $U=A(\mathbf{K})$, we can rewrite $C(\mathbf{K})$ in the form

$$
\mathbf{C}(\mathbf{K})=\left\{\varphi\left(C_{I}\right) \mid I \subset\{0, \ldots, d-1\}, \varphi \in U\right\},
$$


and the isomorphism $\mathbf{C}(\mathbf{K}) \rightarrow \mathbf{C}(U)$ is given by

$$
\varphi\left(C_{I}\right) \rightarrow \varphi U_{I} \quad(I \subset\{0, \ldots, d-1\}, \varphi \in U) .
$$

Of particular interest are the barycentric subdivisions of the classical Euclidean regular $d$-polytopes. Here, the well-known geometrically constructed barycentric subdivision is in a sense isomorphic to the corresponding combinatorial barycentric subdivision. In fact, the simplices in the geometrical subdivision are in one-to-one correspondence with the simplices in the combinatorial subdivision, that is, the totally ordered subsets. The vertices of the $d$-dimensional characteristic orthoscheme belonging to the $(d-1)$-simplex $C_{\varnothing}=f \backslash\left\{F_{-1}, F_{d}\right\}$ are just the centers of $F_{d}$ and the faces in $C_{\varnothing}$ (cf. Coxeter [6]). Hence, the geometrical subdivision is the 'pyramid' over the combinatorial subdivision whose apex is the center of $F_{d}$. The Euclidean reflection in the wall opposite to the center of the $i$-face in $f$ corresponds to the combinatorial reflection in the wall $C_{\{i\}}$ of $C_{\varnothing}$, for $i=0, \ldots, d-1$.

These considerations extend also to regular complex polytopes (cf. Coxeter [7]). For regular honeycombs in the spherical, Euclidean and hyperbolic spaces the two subdivisions actually coincide (cf. Coxeter $[\mathbf{5}, \mathbf{6}])$.

In general, the simplicial complex $\mathbf{C}(\mathbf{K})$ will not be an incidence-complex. Since it is a $d$-complex by the results of $\S 3$, the lacking property is (I4). While the numbers $k_{0}, \ldots, k_{d-2}$ of (I4) exist trivially, and are 2, the number $k_{d-1}\left(C_{\{i\}}\right)$ of the $(d-2)$-face $C_{\{i\}}$ depends on $i$ and is given by $k_{d-1}\left(C_{\{i\}}\right)=\left|R_{i}: U_{\varnothing}\right|=$ $\left|R_{i}: R_{-1}\right|=\tilde{k}_{i}(i=0, \ldots, d-1)$, where $\tilde{k}_{0}, \ldots, \tilde{k}_{d-1}$ are the numbers belonging to $\mathbf{K}$. Hence, if $\mathbf{K}$ is homogeneous of order $k-1$, then $\mathbf{C}(\mathbf{K})$ is a nondegenerate incidence-complex with $k_{d-1}=k$.

In particular, if $\mathbf{K}$ is an incidence-polytope, then $\mathbf{C}(\mathbf{K})$ is also an incidencepolytope. In this particular case, $U_{\varnothing}=\{e\}$, and $R_{i}$ is generated by an involutory automorphism $\rho_{i}$ of $\mathbf{K}(i=0, \ldots, d-1)$. The automorphisms $\rho_{0}, \ldots, \rho_{d-1}$ satisfy the relations

$$
\begin{cases}\rho_{i}^{2}=e, & \text { if } 0 \leq i \leq d-1, \\ \left(\rho_{i} \rho_{j}\right)^{2}=e, & \text { if } 0 \leq i<j-1 \leq d-2, \\ \left(\rho_{i} \rho_{i+1}\right)^{p_{i+1}}=e & \text { if } 0 \leq i \leq d-2,\end{cases}
$$

where $\left\{p_{1}, p_{2}, \ldots, p_{d-1}\right\}$ is the type of the incidence-polytope $\mathbf{K}$ (cf. [21, p. 45]).

5. Building blocks. In the construction of dodecahedral tilings for the Euclidean 3-space suitable dissections of the 3-simplex were derived from the 120-cell in 4-space by projection and were used as building blocks for the tilings. These dissections can be regarded as monotypic 4-complexes whose facets are dodecahedra. The 2-faces $F$ of the complex that lie in one of the 2-faces of the 3-simplex can be characterized by the property $k_{3}(F)=1$.

Analogously, if a convex $d$-polytope $Q$ has all its facets isomorphic to some convex $(d-1)$-polytope $P$ and at least one $d$-valent vertex $x$, then the central projection from $x$ onto the $(d-1)$-simplex $T$ spanned by the neighboured vertices of $x$ in $Q$ yields a dissection of $T$ into convex $(d-1)$-polytopes isomorphic to $P$. Again, this dissection can be regarded as a monotypic $d$-complex $B$ of type $P$. The number of its facets is the number of facets of $Q$ minus $d$. For the construction of combinatorial complexes there is clearly no need to map $T$ onto any other $(d-1)$-simplex as in the construction of dodecahedral tilings. 
This construction provides a number of interesting building blocks.

The vertex-figures of the cyclic $2 n$-polytope $C(v, 2 n)$ with $v$ vertices are known to be of the same combinatorial type as the cyclic $(2 n-1)$-polytopes $C(v-1,2 n-1)$ (cf. Perles-Shephard [19], Altshuler-Perles [1]). Therefore, the dual $Q:=(C(v, 2 n))^{*}$ of $C(v, 2 n)$ is a simple (that is, $2 n$-valent) $2 n$-polytope whose facets are isomorphic to the dual $C(v-1,2 n-1)$. In other words, there exist $2 n$-dimensional building blocks $\mathbf{B}$ whose facets are isomorphic to $(C(v-1,2 n-1))^{*}$.

Our considerations are also of some interest in connection with two problems on simple convex polytopes. It is an open problem, whether or not in dimensions $d-1 \geq 5$ each simple $(d-1)$-polytope $P$ is the facet-type of an equifacetted convex $d$-polytope $Q^{\prime}$. For dimensions $d-1 \leq 4$, the answer is in the negative (cf. PerlesShephard [19], Barnette [2]). On the other hand it is also unknown, if each simple $(d-1)$-polytope $P$ that is the facet-type of a $Q^{\prime}$ is also the facet-type of a simple equifacetted $d$-polytope $Q$ (cf. Perles-Shephard [19]). In case this should be true (in fact, the existence of a least one $d$-valent vertex of $Q$ would suffice), then $Q$ would lead to a monotypic building block $\mathbf{B}$ of type $P$.

It is worth mentioning that for purely combinatorial purposes we can always replace the convex $d$-polytope $Q$ by an equifacetted spherical complex in the $(d-1)$ sphere $\mathbb{S}^{d-1}$. Then, however, we have to work with refinements of simplices (in a similar sense as in Grünbaum [13, p. 199]) instead of dissections obtained by projection.

The building blocks obtained by projection are particular examples of face-toface dissections $\mathbf{B}$ of a $(d-1)$-simplex $T$ into convex $(d-1)$-polytopes. Here, the face-to-face property simply means that the intersection of any two polytopes is either empty or a face of each. Hence, $\mathbf{B}$ is a nondegenerate $d$-complex. Also it has the property that the intersection of any face $F$ of $\mathbf{B}$ with a face of $T$ is a face of $F$.

Let $\mathbf{B}$ be such a complex. For the construction of larger complexes from several copies of $\mathbf{B}$ we have some freedom in assigning the walls of $T$ to the generators $R_{i}$ of the group $U$ (see $\S 3$ ). Therefore, assume that the vertices of $T$ are labelled by the numbers $0,1, \ldots, d-1$. These labels are kept fixed from now on. Clearly, the faces of $T$ are in one-to-one correspondence with the subsets of $\{0,1, \ldots, d-1\}$.

For each face $F$ of $\mathbf{B}$, there is a minimal face $H$ of $T$ containing $F$. By the type $t(F)$ of $F$ we mean the subset of $\{0,1, \ldots, d-1\}$ whose elements are the labels of the vertices of $T$ not in $H$. In particular, $t(F)=\varnothing$ if $H=T$, and $t(F)=\{i\}$ if $H$ is the wall of $T$ opposite to the vertex $i$. Obviously, if $F$ and $G$ are faces in $\mathbf{B}$ and $F$ a face of $G$, then $t(G) \subset t(F)$, but not necessarily a proper subset if $F<G$.

We call the pair $(\mathbf{B}, t)$ consisting of a face-to-face dissection $\mathbf{B}$ of a $(d-1)$ simplex into convex $(d-1)$-polytopes and the type-function $t$ on $\mathbf{B}$ a polyhedral $d$-building-block, or briefly, a polyhedral $d$-block.

In $\S 6$ we will also deal with a more general notion of a $d$-block. Therefore, let us assume from now on that $\mathbf{B}$ is an arbitrary nondegenerate $d$-complex with $(-1)$-face $F_{-1}$ of $d$-face $F_{d}$. Suppose that with each face $F$ of $\mathbf{B}$ there is associated a subset $t(F)$ of $\{0, \ldots, d-1\}$, called the type of $F$, such that the following conditions are satisfied.

(T1) $t\left(F_{-1}\right) \neq \varnothing$.

(T2) If $F \leq G$, then $t(G) \subset t(F)$. 
(T3) If $F \neq F_{-1}$ and $I \subset t(F)$, then there is a face $G$ with $F \leq G$ and $t(G)=I$. For each $F$ and each $i$ in $t(F)$, there is a $(d-2)$-face $G$ with $F \leq G$ and $t(G)=\{i\}$.

(T4) If $F, G \leq H \neq F_{d}$, then there exists a face $H^{\prime}$ with $F, G \leq H^{\prime} \leq H$ such that $t\left(H^{\prime}\right)=t(F) \cap t(G)$.

The subset $D(t):=t\left(F_{-1}\right)$ is called the range of $t$. By (T2), $t(F) \subset D(t)$ for all $F$ in B. From the second part of (T3) we can deduce that $t(F)=\varnothing$, if $F=F_{d}$ or $F$ is a facet of $\mathbf{B}$, and that the cardinality of $t(F)$ is at most 1 if $F$ is a $(d-2)$-face. Also, the case $F=F_{-1}$ shows that for each $i$ in $D(t)$ there is a $(d-2)$-face $G$ of $\mathbf{B}$ with $t(G)=\{i\}$.

We call the pair $(\mathbf{B}, t)$ consisting of the nondegenerate $d$-complex $\mathbf{B}$ and the type-function $t$ on $\mathbf{B}$ with range $D=D(t)$ a $d$-building block with range $D$, or briefly, a $d$-block with range $D$. If no confusion can arise, we simply say that $B$ is a $d$-block with range $D$. A $d$-block with range $D=\{0, \ldots, d-1\}$ is simply called a $d$-block. The most interesting examples are obtained for the case where the range is $D=\{0, \ldots, d-1\}$. The reader might find it easier to verify the subsequent results first for this particular case, and leave the details of the generalization to $d$-blocks of smaller range to a second reading.

The polyhedral $d$-blocks are particular examples of $d$-blocks. Here, the typefunction $t$ is induced in a natural way by labelling the vertices of the respective $(d-1)$-simplex. The properties (T1)-(T4) for $t$ are easily checked, and $t$ has even the additional property that $t(F)=\{0, \ldots, d-1\}=D$ if and only if $F=F_{-1}$.

Other examples of $d$-blocks can be obtained as follows. Assume that the $d$ complex $\mathbf{B}$ has $(d-2)$-faces $G_{0}, \ldots, G_{d-1}$ such that the infimum of any two of them is $F_{-1}$. Defining $t\left(F_{-1}\right):=\{0, \ldots, d-1\}, t(F)=\{i\}$ if $F$ is a face of $G_{i}$ different from $F_{-1}(0 \leq i \leq d-1)$, and $t(F):=\varnothing$ for all other faces of $\mathbf{B}$ provides a $d$-block $(\mathbf{B}, t)$.

Let $(\mathbf{B}, t)$ be a $d$-block with range $D$, and $D^{\prime}$ any subset of $\{0, \ldots, d-1\}$ with $D \cap D^{\prime} \neq \varnothing$. Then, $D^{\prime}$ induces a new $d$-block $\left(\mathbf{B}, t^{\prime}\right)$ with range $D \cap D^{\prime}$ defined by $t^{\prime}(F):=t(F) \cap D^{\prime}$ for $F$ in $\mathbf{B}$. If $(\mathbf{B}, t)$ is a polyhedral $d$-block (then, $D=$ $\{0, \ldots, d-1\})$, we call $\left(\mathbf{B}, t^{\prime}\right)$ a polyhedral $d$-block with range $D^{\prime}$.

Furthermore, each $d$-block $(\mathbf{B}, t)$ with range $D$ gives rise to a class of blocks each obtained from $(\mathbf{B}, t)$ by permutations of the index set $\{0, \ldots, d-1\}$. In fact, if $\sigma$ is any permutation of $\{0, \ldots, d-1\}$, then $t_{\sigma}(F):=\sigma(t(F))$ for $F \in \mathbf{B}$ defines a new $d$-block $\left(\mathbf{B}, t_{\sigma}\right)$ with range $\sigma(D)$. This corresponds to the free choice for labelling the vertices of the underlying $(d-1)$-simplex of a polyhedral $d$-block.

Finally, if $(\mathbf{B}, t)$ is a $d$-block with range $D, F$ a face of $\mathbf{B}$ with $t(F) \neq \varnothing$, and $B_{F}:=\{G \mid G \geq F\}$ the coface of $F$ in $\mathbf{B}$, then the restriction $t_{F}$ of $t$ to $\mathbf{B}_{F}$ induces a $(d-1-\operatorname{dim}(F))-\operatorname{block}\left(\mathbf{B}_{F}, t_{F}\right)$ with range $D_{F}:=t(F)$.

6. The gluing process. The last step in our construction is the gluing of several copies of a given $d$-block $(\mathbf{B}, t)$ with range $D, D \subset\{0, \ldots, d-1\}$. This gluing process is provided by a group $U, U=\left\langle R_{i} \mid i \in D\right\rangle$, acting on the respective simplicial complex $\mathbf{C}(U)$ as described in $\S 3$. The copies of the block fit together in such a way that any two neighboured blocks correspond to each other by a 'combinatorial reflection' in their common wall. This leads to a nondegenerate $d$-complex $\mathbf{T}$. 
Our methods cover the construction of tilings of the Euclidean $(d-1)$-space $E^{d-1}$ (for $D=\{0, \ldots, d-1\}$ ) as well as the construction of dissections of regular $(d-1)$-polytopes (for $D=\{0, \ldots, d-2\}$ ) by means of polyhedral $d$-blocks.

From now on let $(\mathbf{B}, t)$ be an arbitrary $d$-block with range $D$ of cardinality $m$, $D \subset\{0, \ldots, d-1\}$. Let $U$ be a group generated by subgroups $R_{i}, i \in D$, that has the properties of $\S 3$, in particular the intersection property (1). The $d$-complex $\mathbf{T}$ is now derived from a suitable equivalence relation on $U \times \mathbf{B}$ describing the gluing process.

DEFINITION. By $\sim$ we denote the equivalence relation on $U \times \mathbf{B}$ defined by

$$
(\varphi, F) \sim\left(\psi, F^{\prime}\right) \leftrightarrow\left\{\begin{array}{l}
F=F^{\prime}=F_{d} \text { and } \varphi, \psi \in U, \text { or } \\
F=F^{\prime} \neq F_{d} \text { and } \psi^{-1} \varphi \in U_{t(F)} .
\end{array}\right.
$$

Let $\mathbf{T}=\mathbf{T}(\mathbf{B} \mid U)$ (or more exactly, $\mathbf{T}\left(\mathbf{B}, t \mid U ; R_{i}, i \in D\right)$ ) be the set of equivalence classes for $\sim$. By $[\varphi, F]$ we denote the equivalence class of the element $(\varphi, F)$ of $U \times \mathbf{B}$. On $\mathbf{T}$ we introduce the partial order $\leq$ defined by

$$
[\varphi, F] \leq\left[\psi, F^{\prime}\right] \leftrightarrow\left\{\begin{array}{l}
F^{\prime}=F_{d} \text { and } \varphi, \psi \in U, \text { or } \\
F \leq F^{\prime} \neq F_{d} \text { and } \psi^{-1} \varphi \in U_{t(F)} .
\end{array}\right.
$$

First we have to check that $\sim$ is an equivalence relation, and that $\leq$ is both well defined and a partial order on $\mathbf{T}$. While the properties for $\sim$ are trivially satisfied, the properties for $\leq$ depend on the condition (T2) for the type-function $t$. In particular, if $F \leq F^{\prime} \neq F_{d},[\varphi, F]=[\tilde{\varphi}, F],\left[\psi, F^{\prime}\right]=\left[\tilde{\psi}, F^{\prime}\right]$ and $\psi^{-1} \varphi \in U_{t(F)}$, then

$$
\tilde{\psi}^{-1} \tilde{\varphi}=\left(\tilde{\psi}^{-1} \psi\right)\left(\psi^{-1} \varphi\right)\left(\varphi^{-1} \tilde{\varphi}\right) \in U_{t\left(F^{\prime}\right)} \cdot U_{t(F)} \cdot U_{t\left(F^{\prime}\right)}=U_{t(F)}
$$

This argument shows that $\leq$ is well defined. The transitivity of $\leq$ follows in a similar fashion. Note that the only equivalence classes belonging to $F_{-1}$ and $F_{d}$ are $\left[e, F_{-1}\right]$ and $\left[e, F_{d}\right]$, respectively. Also $\left[e, F_{-1}\right] \leq[\varphi, F] \leq\left[e, F_{d}\right]$ for all $\varphi$ in $U$ and $F$ in $\mathbf{B}$.

For $\varphi$ in $U$ let $\mathbf{B}_{\varphi}:=\{[\varphi, F] \mid F \in \mathbf{B}\}$. Then, coincidence or incidence of two elements $[\varphi, F]$ and $\left[\varphi, F^{\prime}\right]$ in $B_{\varphi}$ means nothing else than coincidence or incidence of $F$ in $F^{\prime}$ in $\mathbf{B}$, respectively. Therefore, we may regard the subsets $\mathbf{B}_{\varphi}$ for $\varphi \in U$ as the copies of the block $\mathbf{B}$.

Let $\varphi$ and $\psi$ be in $U$, and assume that the $(m-1)$-simplices $\varphi U_{\varnothing}$ and $\psi U_{\varnothing}$ of $\mathbf{C}(U)$ intersect in a face, say $\varphi U_{I}=\psi U_{I}$. Then, the copies $\mathbf{B}_{\varphi}$ and $\mathbf{B}_{\psi}$ of $\mathbf{B}$ are glued along corresponding faces of type $\mathcal{J}$ with $I \subset \mathcal{J}$ (and along their $d$-faces). By properties (T2) and (T3) for $t$, this means gluing along faces of type $I$ and all their faces (and along the $d$-faces).

Furthermore, if $[\varphi, F] \leq\left[\psi, F^{\prime}\right]$ and $F^{\prime} \neq F_{d}$, then $\psi^{-1} \varphi \in U_{t(F)}$ implies $[\varphi, F]=$ $[\psi, F]$. This shows that no element in $\mathbf{B}_{\psi}$ can majorize an element not in $\mathbf{B}_{\psi}$. In other words, $\mathbf{T}$ is endowed with the partial order that preserves incidences in each copy of the block and creates new incidences only via the gluing process.

THEOREM 1. Let $(\mathbf{B}, t)$ be a d-block with range $D$ consisting of a nondegenerate $d$-complex $\mathbf{B}$ and a type-function $t$ on $\mathbf{B}$ with range $D, D \subset\{0, \ldots, d-1\}$. Let $U=\left\langle R_{i} \mid i \in D\right\rangle$ be a group whose generating subgroups $R_{i}$ satisfy the intersection property (1) of $\S 3$. Then, the partially ordered set $\mathbf{T}=\mathbf{T}(\mathbf{B} \mid U)$ has the following properties. 
(a) $\mathbf{T}$ is a nondegenerate d-complex. $\mathbf{T}$ is finite, if and only if $\mathbf{B}$ and the index $\left|U: U_{\varnothing}\right|$ are finite.

(b) The group $U$ operates on $\mathbf{T}$ and induces a group of automorphisms of $\mathbf{T}$. The number of transitivity classes into which the set of all facets of $\mathbf{T}$ is dissected equals the number of facets in $\mathbf{B}$. Each facet in $\mathbf{B}$ represents exactly one class.

(c) If $\mathbf{B}$ is monotypic of type $\mathbf{L}$ (where $\mathbf{L}$ is a $(d-1)$-complex), then $\mathbf{T}$ is also monotypic of type $\mathbf{L}$.

(d) Let $F$ be a face in $\mathbf{B}, F \neq F_{d}, \mathbf{B}_{F}$ its coface in $\mathbf{B}, t_{F}$ the restriction of $t$ to $\mathbf{B}_{F}, D_{F}:=t(F)$, and $\varphi$ in $U$. Then, the coface in $\mathbf{T}$ of the face $[\varphi, F]$ of $\mathbf{T}$ is isomorphic to $\mathbf{B}_{F}$ if $D_{F}=\varnothing$, or to the $(d-1-\operatorname{dim}(F))$-complex $\mathbf{T}\left(\mathbf{B}_{F} \mid U_{D_{F}}\right)=$ $\mathbf{T}\left(\mathbf{B}_{F}, t_{F} \mid U_{D_{F}} ; R_{i}, i \in D_{F}\right)$ if $D_{F} \neq \varnothing$. In particular, the number of isomorphism types of vertex-figures in $\mathbf{T}$ is finite if the corresponding number for $\mathbf{B}$ is finite.

(e) For each $(d-2)$-face $[\varphi, F]$ in $\mathbf{T}$, the number $k_{d-1}(\mathbf{T},[\varphi, F])$ of facets of $\mathbf{T}$ surrounding $[\varphi, F]$ is given by

$$
k_{d-1}(\mathbf{T},[\varphi, F])= \begin{cases}k_{d-1}(\mathbf{B}, F), & \text { if } t(F)=\varnothing, \\ \left|R_{i}: U_{\varnothing}\right| k_{d-1}(\mathbf{B}, F), & \text { if } t(F)=\{i\} .\end{cases}
$$

ProOF. The group $U$ acts on $\mathbf{T}$ in a natural manner. Each $\tau$ in $U$ induces the automorphism $\tilde{\tau}: \mathbf{T} \rightarrow \mathbf{T}$ defined by $\tilde{\tau}([\varphi, F])=[\tau \varphi, F]$ for $\varphi \in U$ and $F \in \mathbf{B}$. Here, $\tilde{\tau}$ is the identity, if and only if $\tau \in \varphi U_{t(F)} \varphi^{-1}$ for all $\varphi$ and $F$, that is, $\tau \in \varphi^{-1} U_{\varnothing \varphi}$ for all $\varphi$. Clearly, for each $F$ in $\mathbf{B}, U$ acts transitively on the faces $[\varphi, F]$ for $\varphi \in U$. This shows in particular that the coface of a face $[\varphi, F]$ in $\mathbf{T}$ is isomorphic to the coface $\mathbf{T}_{F}$ of $[e, F]$ in $\mathbf{T}$.

Now, let $F$ be a face in $B$ different from $F_{d}, t_{F}$ the restriction of $t$ to the coface $\mathbf{B}_{F}$ of $F$ in $\mathbf{B}$, and $D_{F}:=t(F)$. By definition, $[e, F] \leq\left[\psi, F^{\prime}\right]$ in $\mathbf{T}$, if and only if $F^{\prime}=F_{d}$, or $F_{d} \neq F^{\prime} \in \mathbf{B}_{F}$ and $\psi \in U_{t(F)}$. Now, two cases are possible.

If $D_{F}=\varnothing$, then $\psi \in U_{D_{F}}=U_{\varnothing}$ and $F \leq F^{\prime} \neq F_{d}$ in $B$ imply $\left[\psi, F^{\prime}\right]=\left[e, F^{\prime}\right]$. This proves isomorphism of $\mathbf{T}_{F}$ and $\mathbf{B}_{F}$.

If $D_{F} \neq \varnothing$, then $t_{F}$ is a type-function on the $(d-1-\operatorname{dim}(F))$-complex $\mathbf{B}_{F}$ with range $D_{F}$. Then, we can show isomorphism of $\mathbf{T}_{F}$ and the partially ordered set $\mathbf{T}\left(\mathbf{B}_{F} \mid U_{D_{F}}\right)$ as follows. As already mentioned, the faces of $\mathbf{T}_{F}$ are exactly the faces $\left[\psi, F^{\prime}\right]$ with $F^{\prime} \in \mathbf{B}_{F}$ and $\psi \in U_{D_{F}}$. On the other hand, the equivalence relation defining $\mathbf{T}\left(\mathbf{B}_{F} \mid U_{D_{F}}\right)$ is exactly the restriction to $U_{D_{F}} \times \mathbf{B}_{F}$ of the equivalence relation defining $\mathbf{T}$. In fact, by property (T2) for $t$, we cannot have $\left(\varphi, F^{\prime}\right) \sim$ $\left(\psi, F^{\prime}\right)$ in $U \times \mathbf{B}_{F}, F_{d} \neq F^{\prime} \in \mathbf{B}_{F}$ as well as $\psi \in U_{D_{F}}$ unless $\varphi \in U_{D_{F}}$ and $\psi^{-1} \varphi \in U_{t_{F}\left(F^{\prime}\right)}=U_{t\left(F^{\prime}\right)}$. This establishes a one-to-one correspondence between the elements of $\mathbf{T}_{F}$ and $\mathbf{T}\left(B_{F} \mid U_{D_{F}}\right)$. By a similar argument, the partial orders are the same, so that $\mathbf{T}_{F}$ and $\mathbf{T}\left(\mathbf{B}_{F} \mid U_{D_{F}}\right)$ are actually isomorphic.

In order to show that $\mathbf{T}$ is a $d$-complex we have to check properties (I1)-(I3) for $\mathbf{T}$. While (I1) and (I2) are trivially satisfied (with $\left[e, F_{-1}\right]$ and $\left[e, F_{d}\right]$ as the smallest and greatest elements, respectively), the connectivity property (I3) needs some further considerations.

First we prove that any two flags $f$ and $g$ in $\mathbf{T}$ can be joined by a sequence of flags, each differing from the predecessor in the sequence in at most one face. Obviously, we may assume that $f$ is contained in the copy $\mathbf{B}_{e}$ of $\mathbf{B}$, and $g$ in some copy $\mathbf{B}_{\varphi}=\{[\varphi, F] \mid F \in \mathbf{B}\}$ for $\varphi$ in $U$. Since $U$ is generated by the subgroups $R_{i}$ for $i$ in $D$, we may write $\varphi=\varphi_{1} \varphi_{2} \cdots \varphi_{n}$ with each $\varphi_{k}$ in some $R_{i_{k}}$. By defining 
$\psi_{0}:=e, \psi_{k}:=\varphi_{1} \varphi_{2} \cdots \varphi_{k}$ for $k=1, \ldots, n$ and $\mathbf{B}_{k}:=\mathbf{B}_{\psi_{k}}$ for $k=0, \ldots, n$, we get a sequence $\mathbf{B}_{e}=\mathbf{B}_{0}, \mathbf{B}_{1}, \ldots, \mathbf{B}_{n}=\mathbf{B}_{\varphi}$ of copies of $\mathbf{B}$. For $k=0, \ldots, n-1$, the $(|D|-1)$-simplices $\psi_{k} U_{\varnothing}$ and $\psi_{k+1} U_{\varnothing}$ of $\mathbf{C}(U)$ intersect in the $(|D|-2)$-face $\psi_{k} U_{\left\{i_{k}\right\}}=\psi_{k+1} U_{\left\{i_{k}\right\}}$, so that $\mathbf{B}_{k}$ and $\mathbf{B}_{k+1}$ are glued along corresponding faces of type $\left\{i_{k}\right\}$ and all their faces (and along the $d$-faces). By property (T3) for $t$, there is really a $(d-2)$-face $G_{k}$ of $\mathbf{B}$ such that $t\left(G_{k}\right)=\left\{i_{k}\right\}$; hence $\left[\psi_{k}, G_{k}\right]=\left[\psi_{k+1}, G_{k}\right]$. Taking into consideration the fact that any two flags in the same copy of $\mathbf{B}$ can of course be joined by a suitable sequence of flags all contained in that copy, our arguments prove that the given flags $f$ and $g$ of $\mathbf{T}$ can be joined by a sequence of flags, each differing from the predecessor in at most one face.

But as all cofaces of $\mathbf{T}$ are isomorphic either to some $\mathbf{B}_{F}$ or some $\mathbf{T}\left(\mathbf{B}_{F} \mid U_{D_{F}}\right)$, this property is not only satisfied for $\mathbf{T}$ but also for each coface of $\mathbf{T}$. Together with the fact that the faces of $\mathbf{T}$ different from $\left[e, F_{d}\right]$ are isomorphic to faces of $\mathbf{B}$, and so are connected, this proves the connectivity property (I3) for $\mathbf{T}$. Consequently, $\mathbf{T}$ is a $d$-complex.

The nondegeneracy of $\mathbf{T}$ is now a consequence of propety (T4) for $t$. Obviously, it suffices to show the existence of an infimum in $\mathbf{T}$ for any two nonincident faces of $\mathbf{T}$. By the transitivity properties of $U$, we may restrict the considerations to faces $\left[e, H_{1}\right]$ and $\left[\varphi, H_{2}\right]$ with $H_{1}, H_{2} \in \mathbf{B}$ and $\varphi \in U$. All faces of $\mathbf{T}$ less than or equal to $\left[e, H_{1}\right]$ are of the form $[e, F]$ with $F \leq H_{1}$. By $H$ we denote the infimum of $H_{1}$ and $H_{2}$ in $\mathbf{B}$.

Now, if $[e, F],[e, G] \leq\left[e, H_{1}\right],\left[\varphi, H_{2}\right]$, then $F, G \leq H$ and $\varphi \in U_{t(F)} \cap U_{t(G)}=$ $U_{t(F) \cap t(G)}$. Note that the last equation follows from the intersection property (1) for $U$. Applying (T4) shows the existence of a face $H^{\prime}$ with $F, G \leq H^{\prime} \leq H$ and $\varphi \in U_{t(F) \cap t(G)}=U_{t\left(H^{\prime}\right)}$. But then $[e, F],[e, G] \leq\left[e, H^{\prime}\right] \leq\left[e, H_{1}\right],\left[\varphi, H_{2}\right]$. Note that the first inequality sign is strict, if and only if $[e, F]$ and $[e, G]$ are not incident. As $\mathbf{T}$ is finite dimensional, these considerations prove the existence of an infimum for $\left[e, H_{1}\right]$ and $\left[\varphi, H_{2}\right]$. That establishes the nondegeneracy of $\mathbf{T}$.

The remaining properties are now easily checked.

The finiteness of $\mathbf{T}$ is clearly equivalent to the finiteness of $\mathbf{B}$ and $\mathbf{C}(U)$. But $\mathbf{C}(U)$ is finite, if and only if the index $\left|U: U_{\varnothing}\right|$ is finite. This completes the proof of part (a) of Theorem 1.

It was already mentioned that, for each face $F$ in $\mathbf{B}$, the group $U$ acts transitively on the faces $[\varphi, F]$ for $\varphi$ in $U$. In particular, $[\varphi, F]=\tilde{\varphi}([e, F])$, where $\tilde{\varphi}$ is the automorphism of $\mathbf{T}$ belonging to $\varphi$. On the other hand, two faces $[e, F]$ and $\left[e, F^{\prime}\right]$ are equivalent with respect to $U$, if and only if $F=F^{\prime}$. Then, parts (b) and (c) are obvious.

The first statement of (d) was already proved, and the second is an easy consequence of the first. For the proof of (e) we remark that the type of each $(d-2)$-face $F$ in $\mathbf{B}$ is either the empty set or a set with exactly one element. In case $t(F)=\{i\}$ we have $[e, F]<[\psi, G]$, if and only if $F<G$ in $\mathbf{B}$ and $\psi \in U_{\{i\}}=R_{i}$. But two facets $[\psi, G]$ and $\left[\psi^{\prime}, G^{\prime}\right]$ coincide, if and only if $G=G^{\prime}$ and $\psi^{-1} \psi^{\prime} \in U_{t(G)}=U_{\varnothing}$. This proves (e) for the case $t(F)=\{i\}$. As the case $t(F)=\varnothing$ is trivial, the proof of Theorem 1 is now complete.

REMARK 1. Theorem 1 is of particular interest in case $U$ is the automorphism group of a regular incidence-complex $\mathbf{K}$ generated by its natural system of generating subgroups (see $\S 4$ ). Then, $U$ is isomorphic to a group of automorphisms of $\mathbf{T}$. 
In fact, the subgroups $\varphi^{-1} U_{\varnothing} \varphi$ are stabilizers of flags of $\mathbf{K}$, and an automorphism of $\mathbf{K}$ keeps all flags of $\mathbf{K}$ fixed, if and only if it is trivial.

With the help of Theorem 1 we are now able to construct interesting combinatorial complexes whose facets are convex polytopes of the same combinatorial type.

COROLlARY. Let $(\mathbf{B}, t)$ be a polyhedral d-block with range $D=\{0, \ldots, m-1\}$ for one $m$ with $m \leq d$. Furthermore, let $U=\left\langle\rho_{0}, \rho_{1}, \ldots, \rho_{m-1}\right\rangle$ be the automorphism group of a regular $m$-incidence-polytope $\mathbf{K}$ generated by its natural system of involutory generators (here, $R_{i}=\left\langle\rho_{i}\right\rangle$ for $i=0, \ldots, m-1$ ). Then, $\mathbf{T}(\mathbf{B} \mid \mathbf{K}):=\mathbf{T}(\mathbf{B} \mid U)$ is a nondegenerate $d$-complex with only finitely many isomorphism types of facets and vertex-figures. In particular, if $\mathbf{B}$ is monotypic of type $P$ (where $P$ is a convex $(d-1)$-polytope), then $\mathbf{T}(\mathbf{B} \mid \mathbf{K})$ is also monotypic of type $P . \mathbf{T}(\mathbf{B} \mid \mathbf{K})$ is finite if and only if $\mathbf{K}$ is finite. Moreover, $\mathbf{T}(\mathbf{B} \mid \mathbf{K})$ is a d-incidence-polytope, if and only if $m=d$.

PrOOF. Since $\mathbf{K}$ is an incidence-polytope, the stablizer $U_{\varnothing}$ of a flag is trivial. By Theorem $1(\mathrm{e})$, this shows $k_{d-1}(\mathrm{~T},[\varphi, F]) \leq 2$ for all $(d-2)$-faces $[\varphi, F]$ of $\mathbf{T}:=\mathbf{T}(\mathbf{B} \mid \mathbf{K})$. Here, equality holds for all $(d-2)$-faces of $\mathbf{T}$, if and only if each $(d-2)$-face of $\mathbf{B}$ in the boundary of the underlying $(d-1)$-simplex of $\mathbf{B}$ is of nonempty type, that is, $m=d$. As the faces of $\mathbf{T}$ are isomorphic to convex polytopes, the numbers $k_{0}, \ldots, k_{d-2}$ of (I4) are trivially 2 , so that the proof is now complete.

REMARK 2. (a) Let $\mathbf{B}, U$ and $\mathbf{T}$ be as in Theorem $1, D^{\prime}$ a nonempty subset of $D$, and $t^{\prime}$ the type-function on $\mathbf{B}$ with range $D^{\prime}$ defined by $t^{\prime}(F):=t(F) \cap D^{\prime}$ for $F$ in $\mathbf{B}$. Furthermore, we define the subset $T^{\prime}$ of $\mathbf{T}$ by $\mathbf{T}^{\prime}=\left\{[\varphi, F] \mid \varphi \in U_{D^{\prime}}, F \in \mathbf{B}\right\}$. Then, one can easily prove that $\mathbf{T}^{\prime}$ (endowed with the partial order of $\mathbf{T}$ ) is a $d$ complex isomorphic to $\mathbf{T}\left(\mathbf{B}, t^{\prime} \mid U_{D^{\prime}} ; R_{i}, i \in D^{\prime}\right)$. The transforms of $\mathbf{T}^{\prime}$ under the group-action of $U$ on $\mathbf{T}$ correspond to the left cosets of $U_{D^{\prime}}$ in $U_{D}$; two different transforms never intersect in faces belonging to faces of type $\varnothing$ in $\mathbf{B}$.

(b) Let $\mathbf{B}, \mathbf{K}, U$ and $\mathbf{T}=\mathbf{T}(\mathbf{B} \mid \mathbf{K})$ be as in the corollary to Theorem 1 . If $i<m$ and $D^{\prime}:=\{0, \ldots, m-1\} \backslash\{i\}$, then the application of part (a) shows that the $i$-faces of $\mathbf{K}$ are in one-to-one correspondence with the transforms of $\mathbf{T}^{\prime}$ by $U$. Furthermore, $\mathbf{T}^{\prime}$ (and all its transforms) is decomposable into $d$-complexes, each isomorphic to the $d$-complex $\mathbf{T}\left(\mathbf{B} \mid \mathbf{K}_{i}\right)$ that belongs to the $i$-incidence-polytope $\mathbf{K}_{i}$ representing the isomorphism type of the $i$-faces of $\mathbf{K}$.

The corollary is of particular interest in the case where $\mathbf{K}$ is one of the universal regular $m$-incidence-polytopes $\left\{p_{1}, \ldots, p_{m-1}\right\}$ defined below (cf. [21]). Its group is the Coxeter-group with linear Coxeter-diagram

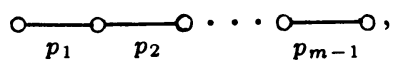

that is, the group $W$ generated by elements $r_{0}, \ldots, r_{m-1}$ and abstractly defined by

$$
\begin{cases}r_{i}^{2}=e, & \text { if } 0 \leq i \leq m-1, \\ \left(r_{i} r_{j}\right)^{2}=e, & \text { if } 0 \leq i<j-1 \leq m-2, \\ \left(r_{i} r_{i+1}\right)^{p_{i+1}}=e, & \text { if } 0 \leq i \leq m-2\end{cases}
$$

(cf. Coxeter-Moser [8]). Here, the elements $r_{0}, \ldots, r_{m-1}$ play the role of $\rho_{0}, \ldots, \rho_{m-1}$ for $U=W$. In other words, writing $W^{i}:=\left\langle r_{k} \mid k \neq i\right\rangle$ for $i=-1,0, \ldots, m$, we can 
regard $\left\{p_{1}, \ldots, p_{m-1}\right\}$ as the set of all left cosets $\varphi W^{i}(\varphi \in W ; i=-1,0, \ldots, m)$, where $\varphi W^{i} \leq \psi W^{j}$ if and only if $\psi^{-1} \varphi \in\left\langle r_{0}, \ldots, r_{j-1}\right\rangle \cdot\left\langle r_{i+1}, \ldots, r_{d-1}\right\rangle$. The incidence-polytopes $\left\{p_{1}, \ldots, p_{m-1}\right\}$ were originally introduced by Tits (cf. [24]) and later by the author (cf. [20]). For a graph-theoretic approach see also Vince [26]. The regular tessellations of spherical, Euclidean and hyperbolic space give particular examples of incidence-polytopes $\left\{p_{1}, \ldots, p_{m-1}\right\}$.

It was proved in [21] that each incidence-polytope $\left\{p_{1}, \ldots, p_{m-1}\right\}$ is universal in the sense that each regular $m$-incidence-polytope $\mathbf{K}$ of type $\left\{p_{1}, \ldots, p_{m-1}\right\}$ can be obtained from it by making suitable identifications. The identifications are provided by the unique homomorphism $\Phi: W=\left\langle r_{0}, \ldots, r_{m-1}\right\rangle \rightarrow U=A(\mathbf{K})=$ $\left\langle\rho_{0}, \ldots, \rho_{m-1}\right\rangle$ mapping $r_{i}$ onto $\rho_{i}(i=0, \ldots, m-1)$.

These facts have some important consequences for the complexes $\mathbf{T}(\mathbf{B} \mid \mathbf{K})$ of our corollary. It turns out that the $d$-complexes $\mathbf{T}\left(\mathbf{B} \mid\left\{p_{1}, \ldots, p_{m-1}\right\}\right)$ belong to the universal $m$-incidence-polytopes $\left\{p_{1}, \ldots, p_{m-1}\right\}$ are universal in a similar sense.

THEOREM 2. Let $(\mathbf{B}, t)$ be a polyhedral d-block with range $D=\{0, \ldots, m-1\}$ for one $m$ with $m \leq d$, and $\mathbf{K}$ be a regular $m$-incidence-polytope of type $\left\{p_{1}, \ldots, p_{m-1}\right\}$ with group $U=A(\mathbf{K})=\left\langle\rho_{0}, \ldots, \rho_{m-1}\right\rangle$. The, the d-complex $\mathbf{T}(\mathbf{B} \mid \mathbf{K})$ can be obtained from $\mathbf{T}\left(\mathbf{B} \mid\left\{p_{1}, \ldots, p_{m-1}\right\}\right)$ by suitable identifications.

PROOF. Let $\Phi: W \rightarrow U$ be the unique homomorphism with $\Phi\left(r_{i}\right)=\rho_{i}$ for $i=0, \ldots, m-1$. For each nonempty subset $I$ of $\{0, \ldots, m-1\}$, we write $W_{I}:=$ $\left\langle r_{i} \mid i \in I\right\rangle$; also $W_{\varnothing}:=\{e\}$. Clearly, $\Phi\left(W_{I}\right)=U_{I}$ for each $I$.

Consider the map

$$
\begin{aligned}
\pi: \mathbf{T}\left(B \mid\left\{p_{1}, \ldots, p_{m-1}\right\}\right) & \rightarrow \mathbf{T}(\mathbf{B} \mid \mathbf{K}) \\
{[w, F] } & \rightarrow[\Phi(w), F] \quad(w \in W, F \in \mathbf{B}) .
\end{aligned}
$$

Then, $\pi$ is well defined, is surjective, and maps incident faces onto incident faces. However, this shows that the complex belonging to $\mathbf{K}$ can be thought of as being obtained from the complex belonging to $\left\{p_{1}, \ldots, p_{m-1}\right\}$ by identifying faces via the map $\pi$, that is, two faces are identified if and only if they have the same image under $\pi$.

REMARK 3 A similar theorem holds also in the more general case, where $W$ is an arbitrary Coxeter-group (not necessarily with a linear Coxeter-diagram) and $U$ a factor-group of $W$ satisfying the intersection property (1). Also, there is no need to restrict attention to polyhedral blocks. This restriction was made for geometrical purposes (see $\S 7$ ).

7. Geometric realizations. In this section we describe a geometrical realization for the complexes $\mathbf{T}(\mathbf{B} \mid \mathbf{K})$ of the corollary to Theorem 1 . In view of Theorem 2 this involves analysis of the universal complexes belonging to Coxeter-groups. We will see that the geometric representation of Coxeter-groups (cf. Bourbaki [3]) leads to a geometrical representation for the respective complexes.

Let $\mathbf{C}$ be a simplicial complex and $x$ be any element not in $\mathbf{C}$. By a 1-fold pyramid (or simply, a pyramid) over $\mathbf{C}$ with apex $x$ we mean the simplicial complex whose vertices are $x$ and the vertices of $\mathbf{C}$, and whose simplices are all sets of the form $F$ or $F \cup\{x\}$ where $F$ is a simplex of $\mathbf{C}$. A $k$-fold pyramid over $\mathbf{C}$ is a pyramid over a $(k-1)$-fold pyramid over $\mathbf{C}(k \geq 2)$. For convenience, we define a 0 -fold pyramid over $\mathbf{C}$ to be $\mathbf{C}$ itself. In the sequel, we make use of the fact that each 
automorphism of a simplicial complex $\mathbf{C}$ can be regarded as an automorphism of any $k$-fold pyramid over $\mathbf{C}$ keeping fixed all vertices not in $\mathbf{C}$.

It is a well-known fact that each finite simplicial complex $\mathbf{C}$ admits a geometrical realization in some finite-dimensional Euclidean space, thereby determining a topological space. If $\mathbf{C}$ has exactly $n$ vertices, then $\mathbf{C}$ can be realized as a subcomplex of the boundary complex of a regular $(n-1)$-simplex $T^{n-1}$ in $E^{n-1}$. Then, each automorphism of $\mathbf{C}$ is induced by a symmetry of $T^{n-1}$, hence by an affine map of $E^{n-1}$.

In particular, these considerations apply to the case where $\mathbf{C}$ is a $k$-fold pyramid over the barycentric subdivision $\mathbf{C}(\mathbf{K})=\mathbf{C}(U)$ of a regular $m$-incidence-polytope $\mathbf{K}$ with group $U=A(\mathbf{K})=\left\langle\rho_{0}, \ldots, \rho_{m-1}\right\rangle$. Here, $n$ equals $k$ plus the number of faces of $\mathbf{K}$ different from the (-1)- and $m$-faces.

Now, let $(\mathbf{B}, t)$ be a polyhedral $d$-block with range $D=\{0, \ldots, m-1\}, m \leq d$, and let $k:=d-m$. Then, we can realize the complex $\mathbf{T}:=\mathbf{T}(\mathbf{B} \mid \mathbf{K})$ of the corollary of Theorem 1 as follows. First note that the maximal simplices of the $k$-fold pyramid $\mathbf{C}$ over $\mathbf{C}(\mathbf{K})$ are now $(d-1)$-dimensional. Therefore, if we implant one copy of the polyhedral block into one of the $(d-1)$-simplices $C$ of $\mathbf{C}$, then the images of this copy under the group $U$ fit together in exactly the same way as the several copies of the block in the construction of $\mathbf{T}$. However, the implanting must be done according to the following rule. For $i=0, \ldots, m-1$, the wall of the block containing the $(d-2)$-faces of type $\{i\}$ must be opposite to the vertex of $C$ representing an $i$-face in $\mathbf{K}$. Consequently, each wall of the block containing only $(d-2)$-faces of type $\varnothing$ is opposite to a vertex of $C$ not in $\mathbf{C}(\mathbf{K})$ (that is, to one of the apices of $\mathbf{C}$ ). Our rule ensures that, for each subset $I$ of $\{0, \ldots, m-1\}$, the group $U_{I}$ keeps exactly that face of $C$ fixed, which is spanned by the apices of $\mathbf{C}(\mathbf{K})$ and the vertices representing $j$-faces of $\mathbf{K}$ with $j \notin I$. This face of $C$ is opposite to that face of $C$ spanned by the vertices representing $j$-faces of $\mathbf{K}$ with $j \in I$. These facts prove that we really obtain a geometrical realization of $\mathbf{T}(\mathbf{B} \mid \mathbf{K})$.

However, this realization is not satisfactory, since the dimension $n-1$ is both high, and not only dependent on the dimension of $\mathbf{C}$ but also on the number of vertices of $\mathbf{C}$.

We can overcome this problem at least for the universal $d$-complexes

$$
\mathbf{T}\left(\mathbf{B} \mid\left\{p_{1}, \ldots, p_{m-1}\right\}\right) .
$$

They admit geometric realizations of both the complex in $E^{d}$ and the respective group $W$ as a subgroup of the general linear group $G L(d)$ of $E^{d}$. With respect to Theorem 2, this gives also a kind of representation of $d$-complexes derived from arbitary regular incidence-polytopes of type $\left\{p_{1}, \ldots, p_{m-1}\right\}$.

We make use of the fact that Coxeter-groups $W=\left\langle\tau_{0}, \ldots, \tau_{m-1}\right\rangle$ (with linear Coxeter-diagram) can be represented as subgroups of the general linear group $G L(m)$ of the real $m$-space. For special cases this representation was discovered in Coxeter [4] and Witt [28]; the general case is due to Tits (cf. [24] or Bourbaki [3, Chapitre 5]).

Via this representation the group $W$ operates on a system $\hat{\mathbf{C}}$ of $m$-dimensional simplicial cones with apex 0 in $E^{m}$. The generators $r_{0}, \ldots, r_{m-1}$ correspond to certain affine reflections in the walls of some fixed $m$-cone $\hat{C}$. This cone $\hat{C}$ is a fundamental region for $W$ in the union of all cones. 


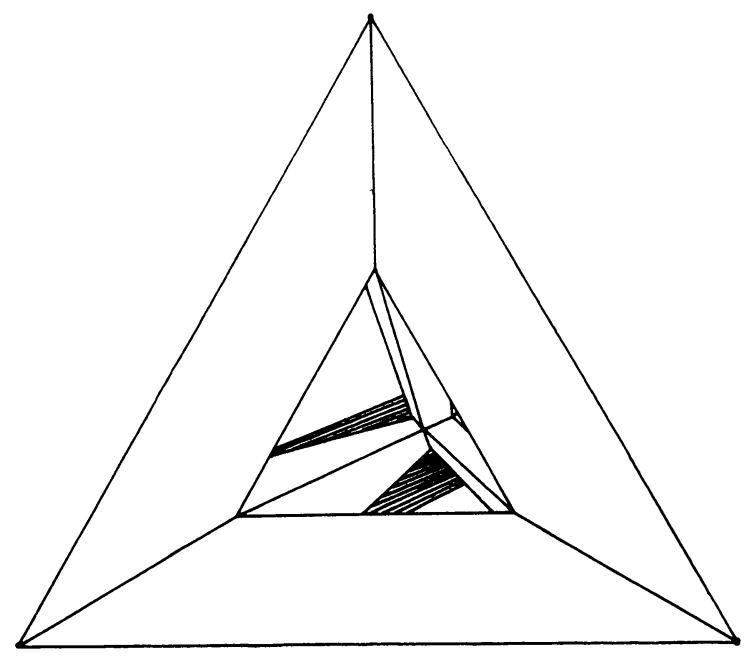

FIgURE 2. The semiplatonic solid $\{4,6 ; 6\}$ found by Wills is bounded by a polyhedral 2-manifold of genus 6 , whose 30 facets are quadrangles, 6 surrounding each of the 20 vertices

Cutting the $m$-cone $\hat{C}$ by a suitable hyperplane of $E^{m}$ not containing 0 we obtain an $(m-1)$-simplex $C^{\prime}$ in $E^{m}$. Now, taking all transforms of $C^{\prime}$ under the action of $W$ on the cone-system gives a simplicial complex in $E^{m}$, where each $(m-1)$ simplex represents exactly one cone of the system. This complex is a geometric realization of the simplicial complex $\mathbf{C}(W)$ belonging to $W$. Also, $W$ operates on it as a subgroup of $G L(m)$, and $C^{\prime}$ is a fundamental region for $W$.

Again we consider a $k$-fold pyramid $\mathbf{C}$ over $\mathbf{C}(W)$. Since $\mathbf{C}(W)$ admits a realization in $E^{m}$, we get a realization of $\mathbf{C}$ in $E^{m+k}=E^{d}$. Also, we may regard $W$ as a subgroup of $G L(d)$ keeping all the apices of $\mathbf{C}$ invariant. Now, if we implant again one copy of the block $(\mathbf{B}, t)$ into one $(d-1)$-simplex $C$ of $\mathbf{C}$, again according to the above-mentioned rule, then the images of this copy fit together in the same way as in the gluing process of $\S 6$. In this way we get a geometrical representation for the universal $d$-complexes $\mathbf{T}\left(\mathbf{B} \mid\left\{p_{1}, \ldots, p_{m-1}\right\}\right)$ in $E^{d}$.

We remark that this construction works equally well for Coxeter-groups with nonlinear Coxeter-diagram.

The universal $m$-incidence-polytope $\left\{4,3^{m-1}, 4\right\}$ is isomorphic to the regular tessellation of $E^{m-1}$ by cubes. In the geometric representation of the respective group $W$ in $E^{m}$ one hyperplane in $E^{m}$ is invariant under $W$, and $W$ operates on it in the same way as the symmetry group of the cubical tessellation on $E^{m-1}$ (cf. Bourbaki [3, Chapitre 5]). Therefore, if we choose the $(m-1)$-simplex $C^{\prime}$ in this hyperplane, then all its transforms by $W$ lie in this hyperplane. Consequently, the resulting simplicial complex coincides (in the isometric sense) with the barycentric subdivision of the cubical tessellation. Therefore, if $(\mathbf{B}, t)$ is a polyhedral $d$-block and $m=d$, then the above-mentioned geometrical realization of $\mathbf{T}\left(\mathbf{B} \mid\left\{4,3^{d-3}, 4\right\}\right)$ coincides with the realization which was described in the Introduction.

It is worth mentioning that other types of building-blocks can be used to get further interesting complexes, which are not necessarily incidence-complexes. For instance, we can choose the 4-block $\mathbf{B}$ shown in Figure 2 (together with a typefunction similar to those for polyhedral blocks); here, the interior of the 2-manifold 
is considered as the unique 3-face of $\mathbf{B}$. This block is one of the semiplatonic solids recently studied by Wills (cf. [27]).

\section{REFERENCES}

1. A. Altshuler and M. A. Perles, Quotient polytopes of cyclic polytopes I (structure and characterization), Israel J. Math. 36 (1980), 97-125.

2. D. Barnette, A simple 4-dimensional nonfacet, Israel J. Math. 7 (1969), 16-20.

3. N. Bourbaki, Groupes et algèbres de Lie, Chapitres 4, 5, 6, Actualités Sci. Indust., Hermann, Paris, 1968.

4. H. S. M. Coxeter, The complete enumeration of finite groups of the form $R_{i}^{2}=\left(R_{i} R_{j}\right)^{k_{i j}}=1$, J. London Math. Soc. 10 (1935), 21-25.

5. __ Twisted honeycombs, CBMS Regional Conf. Ser. Math., no. 4, Amer. Math. Soc., Providence, R.I., 1970.

6. __ Regular polytopes, 3rd ed., Dover, New York, 1973.

7. L Regular complex polytopes, Cambridge Univ. Press, Cambridge, 1974.

8. H. S. M. Coxeter and W. O. J. Moser, Generators and relations for discrete groups, 4th ed., Springer, Berlin, 1980.

9. L. Danzer, Regular incidence-complexes and dimensionally unbounded sequences of such. I, Ann. Discrete Math. 20 (1984), 115-127.

10. L. Danzer and E. Schulte, Reguläre Inzidenzkomplexe I, Geom. Dedicata 13 (1982), 295-308.

11. L. Danzer, B. Grünbaum and G. C. Shephard, Does every type of polyhedron tile three-space?, Structural Topology 8 (1983), 3-14.

12. L. Fejes Tóth, Reguläre Figuren, Akad. Kiado, Budapest, 1965.

13. B. Grünbaum, Convex polytopes, Wiley, London, New York and Sydney, 1967.

14. __ Regularity of graphs, complexes and designs, Problèmès Combinatoires et Théorie des Graphes (Colloq. Internat. CNRS, Orsay, 1976), Colloq. Internat. CNRS, 260, CNRS, Paris, 1976, pp. 191-197.

15. B. Grünbaum and G. C. Shephard, Tilings and patterns, Freeman, San Francisco, Calif., 1987.

16. B. Grünbaum, P. Mani-Levitska and G. C. Shephard, Tiling three-dimensional space with polyhedral tiles of a given isomorphism type J. London Math. Soc. (2) 29 (1984), 181-191.

17. D. G. Larman and C. A. Rogers, Durham symposium on the relations between infinite-dimensional and finite-dimensional convexity, Bull. London Math. Soc. 8 (1976), 1-33.

18. P. McMullen and G. C. Shephard, Convex polytopes and the upper-bound conjecture, London Math. Soc. Lecture Notes Ser, 3, 1971.

19. M. A. Perles and G. C. Shephard, Facets and nonfacets of convex polytopes, Acta Math. 119 (1967), 113-145.

20. E. Schulte, Reguläre Inzidenzkomplexe, Dissertation, Dortmund, 1980.

21. __ Reguläre Inzidenzkomplexe II, Geom. Dedicata 14 (1983), 33-56.

22. _ The existence of nontiles and nonfacets in three dimensions, J. Combin. Theory $\mathbf{A} 38$ (1985), 75-81.

23. _ Nontiles and nonfacets for the Eucildean space, spherical complexes and convex polytopes, J. Reine Angew. Math. 352 (1984), 161-183.

24. J. Tits, Groupes et géométries de Coxeter, Notes polycopiées, I.H.E.S., Paris, 1961.

25. __ Buildings of spherical type and finite BN-pairs, Springer, Berlin, 1974.

26. A. Vince, Regular combinatorial maps, J. Combin. Theory Ser. B 35 (1983), 256-277.

27. J. M. Wills, Semi-platonic manifolds, Convexity and its Applications, edited by P. Gruber and J. M. Wills, Birkhäuser, Basel and Boston, Mass., 1983, pp. 413-421.

28. E. Witt, Spiegelungsgruppen und Aufzählung halbeinfacher Liescher Ringe, Abh. Math. Sem. Univ. Hamburg 14 (1941), 289-322.

Department of Mathematics, GN-50, University of Washington, Seattle, WASHINGTON 98195

Current address: Department of Mathematics, Massachusetts Institute of Technology, Cambridge, Massachusetts 02139 